\title{
Grupo Trilhas: Repensando a trajetória de cuidados
}

\author{
Trillhas group: Rethinking the trajectory of care \\ Grupo Trilhas: Repensando la trayectoria de cuidados
}

\section{Resumo}

O presente trabalho tem como objetivo relatar a experiência do Grupo Trilhas, realizado como intervenção durante a disciplina "Estágio Específico em Psicologia e Saúde" na Universidade Federal do Paraná. O grupo foi realizado em um Centro de Atenção Psicossocial III no estado do Paraná e teve como objetivo discutir os itinerários terapêuticos das/os usuárias/os, ou seja, os caminhos de cuidado percorridos pela rede de saúde ou outros serviços, e favorecer maiores níveis de autonomia em seus percursos futuros. As atividades do grupo foram divididas em quatro eixos: "história de vida", "a rede intersetorial", "construindo a rede de apoio" e "ida ao território" que constituíram dezessete encontros semanais. $\mathrm{O}$ grupo permitiu às/aos usuárias/os uma reflexão sobre suas histórias de vida e a identificação dos serviços resolutivos às suas necessidades em saúde. Além disso, buscou-se fomentar o desenvolvimento de novos interesses em atividades de esporte, cultura, educação e lazer no bairro ou comunidade ao qual os indivíduos estão inseridos, visando expandir sua rotina dentro do território, além do Centro de Atenção Psicossocial.

Palavras-chave: Saúde mental; Determinação social da saúde; Autonomia; Serviços comunitários de saúde mental.

\begin{abstract}
This article aims to present the experience of the group "Trilhas", conducted as an intervention during the discipline "Estágio Específico em Psicologia e Saúde" at the Federal University of Paraná. The group was developed at a Centro de Atenção Psicossocial in the state of Paraná and aimed to identify the patients therapeutic itineraries, that is, the paths taken by de patients during the search for attention for their health conditions and promote greater levels of autonomy in their future paths. The group's proposal was divided into three parts: "life story", "the intersectorial network" and "building the support network" which constituted the seventeen weekly meetings. The group allowed the participants to think about their life stories and to identify the services that answer their health needs. The importance of the group as an instrument that enhances development Sewas observed, providing the development of skills and potential that were previously denied to the participants. In addition, the group owes the mental health service users a reflection on their life stories, identity and foster the development of new interests in sports, culture, education and recreation activities within their neighborhood or community, seeking to expand their routine outside the CAPS.
\end{abstract}

Keywords: Mental health; Social determination of health; Autonomy; Community mental health services.

\section{Resumen}

Este estudio tuvo como objetivo realizar un relato de experiencia del grupo "Trilhas", realizado como intervención durante el curso "Pasantía Específica en Psicología y Salud" en la Universidad Federal de Paraná. El grupo se llevó a cabo en un Centro de Atención Psicosocial III del estado del Paraná y tuvo como objetivo identificar los itinerarios terapéuticos de los usuarios, es decir, las trayectorias de atención tomadas por la red de salud y los demás servicios, favoreciendo mayores niveles de autonomía en sus trayectorias futuras. La propuesta del grupo se dividió en tres partes: "historia de vida", "la red intersectorial" y "construyendo la red de apoyo" que constituyeron las diecisiete reuniones semanales. El grupo permitió a los usuarios reflexionar sobre sus historias de vida e identificar servicios que responden sus necesidades de salud. Se observó la importancia del grupo como instrumento que potencia el desarrollo, brindando el desarrollo de habilidades y potencialidades que antes le eran negadas a los individuos. Además, el grupo permitió a los usuarios reflexionar sobre sus historias de vida, identidad y buscó fomentar el desarrollo de nuevos intereses en el 
deporte, la cultura, la educación y las actividades de ocio dentro de su barrio o comunidad, buscando expandir su rutina fuera del Centro de Atención Psicosocial.

Palabras clave: Salud mental; Determinación social de la salud; Autonomía; Servicios comunitarios de salud mental.

\section{Introdução}

O movimento pela reforma psiquiátrica brasileira surgiu no fim da década de 1970, no contexto da redemocratização do país, tendo como objetivo substituir o cuidado de saúde mental, centrado no hospital psiquiátrico, por um cuidado sustentado em dispositivos diversificados, abertos e de natureza comunitária ou territorial. O movimento foi constituído não apenas como crítica ao subsistema nacional de saúde mental, mas também como uma crítica estrutural à psiquiatria e teve como marca fundamental o reclame da cidadania do louco (Amarante, 2007; Amarante \& Nunes, 2018; Tenório, 2002).

A chamada Lei da Reforma Psiquiátrica, Lei 10.216 (Brasil, 2001), que dispõe sobre a proteção e os direitos das pessoas portadoras de transtornos mentais e redireciona o modelo assistencial em saúde mental, foi sancionada no mesmo ano da III Conferência Nacional de Saúde Mental do Movimento da Luta Antimanicomial, em 2001. Mas apenas no ano de 2011 foi instituída a Rede de Atenção Psicossocial (RAPS) por meio da Portaria GM/MS nº 3.088 de 2011 (Brasil, 2011; Brasil, 2017), possibilitando uma nova dimensão ao conjunto das ações em saúde mental no Sistema Único de Saúde (SUS) com a articulação em rede dos dispositivos no território. Seus principais objetivos são: ampliação do acesso à atenção psicossocial da população, em seus diferentes níveis de complexidade; promoção do acesso das pessoas com transtornos mentais e com necessidades decorrentes do uso do crack, álcool e outras drogas e suas famílias aos pontos de atenção; garantia da articulação e integração dos pontos de atenção das redes de saúde no território, qualificando o cuidado por meio do acolhimento, do acompanhamento contínuo e da atenção às urgências (Brasil, 2011; Brasil, 2017).

Neste contexto, o modelo de atenção psicossocial emerge para romper com a realidade asilar e com a cultura da violência e dar espaço e visibilidade para o sujeito por meio da territorialização do cuidado e da construção dos serviços em espaços abertos, de modo que contribuam para o exercício de cidadania e inclusão social para que aquele que antes era considerado como louco, passe a ser visto como cidadão de direitos (Amarante, 1995; Tenório, 2002).

Segundo Souza e Zambenedetti (2018), a análise dos itinerários terapêuticos permite localizar as potencialidades e os desafios, tanto dos dispositivos de saúde, como da singularidade dos sujeitos e na dimensão social ampliada na trajetória de cuidado das pessoas. O conceito de Itinerários Terapêuticos designa os processos e práticas pelos quais um sujeito perpassa em busca de atendimento e resolução de suas demandas de saúde (Alves, 2015; Alves 2015b; Gerhardt, 2006). No contexto das pessoas em sofrimento psíquico, é necessário um olhar a estes caminhos percorridos em busca de cuidado devido à importância deste conhecimento para a garantia de políticas públicas de qualidade em saúde mental e para que os sujeitos tenham suas necessidades em saúde atendidas pelos dispositivos adequados. Dessa forma, o presente trabalho tem como objetivo relatar a experiência do Grupo Trilhas, que foi desenvolvido como intervenção durante a disciplina "Estágio Específico em Psicologia e Saúde" da Universidade Federal do Paraná. A intervenção teve como objetivo identificar e discutir com as/os usuárias/os seus itinerários terapêuticos, ou seja, seus caminhos de cuidado pela rede de saúde ou outros serviços e favorecer maiores níveis de autonomia em seus percursos futuros.

\section{Metodologia}

Este artigo, de abordagem qualitativa, tem como objetivo relatar a experiência do desenvolvimento de um trabalho de intervenção, denominado "Grupo Trilhas", realizado no curso de psicologia da Universidade Federal do Paraná, durante a disciplina "Estágio Específico em Psicologia e Saúde". O Grupo Trilhas foi desenvolvido em uma instituição da Rede Pública de Saúde Mental, em um Centro de Atenção Psicossocial III, durante os meses de março a novembro de 2019. 
As atividades do grupo ocorreram em 17 encontros semanais, com duração de 1h30min e contaram com uma média de oito usuárias/os por encontro. A intervenção foi estruturada em quatro eixos, sendo eles: "história de vida", "a rede intersetorial", "construindo a rede de apoio" e "ida ao território"; e em cada encontro foi utilizada uma técnica de dinâmica de grupo para iniciar a discussão. O objetivo do grupo foi identificar, juntamente às/aos usuárias/os do CAPS, as trajetórias passadas pela rede intersetorial e outras formas de cuidado e tratamento por eles buscadas, visando auxiliar no planejamento de vida futuro e no desenvolvimento de uma consciência social crítica do processo saúde-doença dentro da perspectiva da saúde mental.

\section{Resultados e Discussão}

Tendo em vista a importância da captura da historicidade do sujeito para pensar a própria trajetória terapêutica, o primeiro eixo do grupo buscou retomar a história de vida das/os participantes, explorando assim a totalidade de relações presentes na vida desses sujeitos de forma a entender seus itinerários terapêuticos. Foi realizada a estratégia de mediação da construção de uma linha do tempo de cada um/a dos/as participantes, contendo aspectos de suas histórias e a busca por cuidados em saúde. Quatro temáticas se destacaram na construção da linha do tempo: a história de vida de cada participante, sua relação com o trabalho, a construção do diagnóstico psiquiátrico e o acesso aos serviços de saúde mental.

Singularmente, a maior parte das/os participantes cresceu com pais rígidos, em condições materiais precárias e com relações afetivas familiares empobrecidas. A maior parte das mulheres havia sofrido algum tipo de violência de gênero doméstica, obstétrica, física e/ou psicológica - que repercutiram em sua saúde mental. A violência contra mulher é considerada um problema social e de saúde pública, havendo estreita correlação entre a violência contra as mulheres e o adoecimento psíquico (Santos, 2009). Segundo Oliveira e Jorge (2007), mulheres que experienciam situações de violência ao longo da vida acabam por experimentar danos na saúde, estresse e sofrimento psíquico.

Durante o grupo, notou-se as consequências do empobrecimento das relações humanas em diversas/os usuárias/os. A maioria, pelas condições precárias de vida, encontrava obstáculos para o desenvolvimento de novas atividades, tais como: interesses por atividades físicas, cursos, dentre outras. O principal motivo atribuído a este fato era a imprescindibilidade de suprir as necessidades básicas humanas, como alimentação e moradia. Leontiev (1969) afirma que as condições sociais de vida do indivíduo e o lugar que este assume nas relações com os outros seres humanos têm papel determinante no tipo de necessidades que surgem e suas possibilidades para satisfazê-las. Nesse sentido, cabe destacar que, com a realização dos grupos, foi possível evidenciar que a vida da maioria das/os usuárias/os se encontrava centrada apenas na busca de uma atividade de trabalho, que promovesse uma renda mínima para a satisfação das necessidades básicas, não encontrando nessa rotina possibilidades para o surgimento de novas necessidades ou a realização de novas atividades. Três usuárias/os relataram que essa centralidade das atividades em relação à sobrevivência ocorria desde a infância. Estes, ainda crianças, trabalhavam na roça para ajudar no sustento da família. Quando questionados sobre esse trabalho, afirmaram que as condições eram precárias e que recebiam apenas o suficiente para a alimentação. Identificou-se, ainda, que tais condições fizeram também com que muitas/os usuárias/os não chegassem a frequentar a escola ou abandonassem os estudos ainda na infância, o que tem impactos sobre seu processo de desenvolvimento.

Algumas/uns participantes trouxeram em sua história de vida o marco do trabalho no processo de adoecimento. As principais causas atribuídas a este fenômeno citadas pelas/os usuárias/os foram: as situações precárias de trabalho, a existência de poucos direitos trabalhistas e a demissão após a primeira crise no ambiente de trabalho. A maioria das/os participantes abordava as questões do trabalho referentes ao seu passado, pois no momento não se encontravam exercendo atividades remuneradas, seja formal ou informal. Nota-se que, apesar dos avanços suscitados pela reforma psiquiátrica, o status da loucura e a sua consequente marginalização não se transformou por completo. Com o advento do capitalismo, em que a saúde está 
atrelada à eficácia e produtividade, a loucura foi convertida em "doença mental" e foi a ela atribuído o mesmo valor no mundo do trabalho: nenhum (Paranhos-Passos \& Aires, 2013).

Essa marginalização é amplamente vista nos Centro de Atenção Psicossocial (CAPS), onde a maior parte das/os usuárias/os não têm emprego formal, dada a dificuldade de responder às exigências do mercado capitalista (Andrade \& Burali, 2013). Essa realidade é um empecilho para a emancipação e mesmo para a ocupação cidadã dos espaços, já que, ao retirar a possibilidade de inserção no mercado de trabalho dessa população, se restringe profundamente o contato social e o acesso a uma grande parte da produção humana. Assim, a alienação, característica da sociedade capitalista, se mostra como um obstáculo fundamental para o desenvolvimento psíquico dessas/es usuárias/os, bem como fonte de seu sofrimento. Conforme afirmado por Almeida (2018, p. 131) "a atividade, como fundamento do desenvolvimento, quando está afetada por obstruções na dinâmica da vida e produz o adoecimento, afeta também o desenvolvimento". Além da falta de trabalho, que foi amplamente relatada pelas/os usuárias/os como fator adoecedor, evidenciou-se no decorrer dos encontros o fato de que a vida das/os usuárias/os estava restrita apenas às atividades oferecidas pelo CAPS. Entende-se que esta situação limita o desenvolvimento da autonomia desses sujeitos, uma vez que eles não encontram espaços além do CAPS para o desenvolvimento de novas atividades ou para produção de vínculo e trocas sociais com outras pessoas (Pande \& Amarante, 2011).

É perceptível, também, que a grande maioria dos usuários acumula a produção e reprodução de sofrimento psíquico em seu histórico de vida, seja em relação à família, ao trabalho, ou a um histórico de vinculação com as instituições psiquiátricas. As internações eram comuns à grande maioria dos participantes no período anterior à criação do CAPS e foi trazida por eles como produtora de grande sofrimento psíquico, além de ser apontada como tendo pouca resolutividade em suas demandas em saúde mental. A partir da análise dos itinerários terapêuticos e o conhecimento da reforma psiquiátrica, os participantes compreenderam o CAPS como o serviço mais adequado às demandas de saúde mental e a UPA (Unidade de Pronto Atendimento) e as Unidades de Saúde às demandas clínicas.

A própria classificação diagnóstica trazida pelos dispositivos, pautada no modelo manicomial, foi percebida pelas/os usuárias/os como geradora de sofrimento, ao enquadrar uma sintomatologia específica em formas de sentir a vida engessadas, reforçando uma estagnação da pessoa, impedindo-a de estabelecer relações que não sejam a partir de uma posição de "louco" ou “doente". De acordo com Kinoshita (1996, p. 56), “o doente mental passa a ter positividade apenas na sua dimensão de doente, de suporte da doença. Em suma, anula-se qualquer valor da pessoa que o assegura como sujeito social".

O segundo eixo do grupo foi composto pela discussão da reforma psiquiátrica, a apresentação da Rede de Atenção Psicossocial e a construção dos itinerários terapêuticos. Para tanto, foi realizada a leitura da Lei 10.216/2001 (Brasil, 2001) e foi construído uma representação gráfica da estrutura da RAPS e da rede intersetorial, sendo apontados pelas/os usuárias/os os dispositivos de saúde buscados durante as suas trajetórias de vida. Essas/es usuárias/os, que estão imersas/os no serviço e recebem atenção das políticas públicas, desconheciam o histórico do próprio CAPS e da luta antimanicomial, ainda que suas vidas tenham sido marcadas por todas as mudanças na legislação e na prática do cuidado em saúde mental. Durante a apresentação da história da Reforma Psiquiátrica, todas/os participantes afirmaram não ter conhecimento prévio sobre a lei da Reforma Psiquiátrica ou o movimento da luta antimanicomial. Após a leitura da lei, muitas/os afirmaram que não sabiam seus direitos e, dessa forma, estavam impossibilitadas/os de buscá-los.

A construção gráfica da estrutura da RAPS se deu de forma coletiva em uma cartolina, onde todos foram levantando os nomes dos dispositivos de saúde pelos quais já passaram, tais como: Unidade de Saúde, Unidade de Pronto Atendimento (UPA), Hospital Psiquiátrico, Hospital Geral, Consultório na Rua, entre outros. Logo em seguida foram discutidas quais demandas ou queixas são atendidas em cada um destes serviços. Também foi questionado junto às/aos usuárias/os em quais dispositivos 
tiveram suas demandas atendidas e, quando não tiveram suas queixas atendidas, os possíveis motivos, tendo como objetivo a construção singular da rede de dispositivos que atendem as demandas de saúde específicas de cada sujeito.

Em seguida foi trabalhado o reconhecimento da rede pessoal de apoio dos participantes através de duas atividades: o círculo da rede de apoio e o mapa da rede social, fornecidos pelo guia do moderador do grupo GAM - Gestão Autônoma da Medicação (Campos et al, 2014). Nestas atividades, as/os usuárias/os adicionaram ao seu círculo. além das pessoas que fazem parte da sua rede de apoio, os dispositivos do território que já fazem parte de sua rotina, bem como aqueles que poderiam vir a fazer, tais como: Centros de Convivência, Igreja, Praças e Parques, Centros Culturais, entre outros.

Este terceiro eixo do grupo foi desenvolvido baseado no conceito de autonomia, definido por Kinoshita (1996) como a capacidade de um indivíduo produzir novas normas para a sua vida, de acordo com as situações que encontra. Dessa forma, a autonomia não se confunde com independência ou auto suficiência, mas se refere à ampliação da possibilidade de estabelecer novas normas por meio da relação com o maior número possível de pessoas ou atividades. Nesse sentido, o grupo buscou apresentar às/aos usuárias/os a diversidade de equipamentos e recursos existentes no território para suprir as suas necessidades, visando a busca de uma rede de apoio tanto de pessoas como de dispositivos de saúde para além do CAPS. A construção gráfica da rede de serviços possibilitou também a conscientização de necessidades e a busca por novas atividades. Um usuário, por exemplo, percebeu que estava negligenciando aspectos da sua saúde física devido à obesidade e sedentarismo. Apesar da prática de exercício físico ser uma necessidade, o participante até então não tinha tomado consciência da sua importância e, a partir do andamento do grupo, teve a possibilidade de buscar um grupo de caminhada.

Durante a intervenção, notou-se que apenas conhecer os pontos da rede e novos locais de socialização não levava as/os usuárias/os à busca ativa por esses lugares. Este fato pode ser atribuído ao empobrecimento das atividades e realidades sociais dos participantes, que limitava o desenvolvimento de novos interesses, e também pelo receio de discriminação e estigma por ser usuário da saúde mental.

Diante deste cenário, identificou-se a importância da atuação dos profissionais que atuam nos CAPS e, consequentemente, das atividades desenvolvidas pelo grupo na direção de um quarto eixo, com o objetivo de estimular a participação das/os usuárias/os em novas atividades e, consequentemente, o desenvolvimento da autonomia e reinserção social. As estratégias utilizadas com o grupo, para estimular o interesse em novas atividades foram: a realização de um bingo de interesses, a criação de personagens e a ida a espaços do território, escolhidos pelas/os usuárias/os.

Tendo em vista que a função do CAPS é, segundo Paranhos-Passos e Aires (2013), a reabilitação psicossocial e a reinserção social, é importante oferecer à/ao usuária/o possibilidades que o capacitem para a vida em sociedade. Uma das formas para restaurar este valor social é a busca de novas atividades. Partindo das contribuições da psicologia histórico-cultural, considera-se que não nos cabe buscar a gênese do ser humano em entidades abstratas, ideais ou supra-humanas, mas sim nas relações concretas que os indivíduos constroem com os outros e com a sociedade por meio das atividades que realizam (Leontiev, 1969).

Logo, foram realizadas visitas a diferentes locais, escolhidos coletivamente com as/os usuárias/os: uma casa de leitura situada em uma Rua da Cidadania (que é uma sede descentralizada de órgãos públicos da prefeitura com oferta de serviços à população); uma escola do bairro que oferecia cursos técnicos; um Centro Estadual de Educação Básica para Jovens e Adultos (CEEBJA) e Educação de Jovens e Adultos (EJA); um Liceu de Ofícios da prefeitura que oferece diferentes cursos profissionalizantes; um museu de arte; parques; um cinema; e um teatro. Buscou-se assim contemplar espaços de lazer, profissionalização, cultura e esporte.

Notou-se também que a realização do grupo possibilitou uma compreensão dos indivíduos enquanto seres coletivos, na medida em que as/os usuárias/os foram se vinculando, identificando-se e implicando-se nas falas das/os outras/os participantes, 
bem como levantando dúvidas ou apontando contradições. O processo grupal pode potencializar o desenvolvimento do indivíduo, rompendo com uma barreira individualista ou biologicista da compreensão do sofrimento (Lane, 1984; Martins, 2007). Os grupos podem proporcionar às/aos usuárias/os uma ferramenta para a construção de relações com demais pessoas e, também, para desenvolver capacidades e potencialidades que outrora foram negadas a esses indivíduos. Além de possibilitarem local para expressão, escuta e acolhimento (Lappann-Botti \& Labate, 2004), os grupos e oficinas terapêuticas representam um instrumento de ressocialização e inserção das/os usuárias/os quando propõem o pensar e agir coletivos, respeitando a diversidade e subjetividade de cada sujeito participante (Azevedo \& Miranda, 2001).

A atuação no equipamento de saúde mental foi fundamentada nos preceitos da reforma psiquiátrica, visando à busca por autonomia e a reinserção social. Com base em Costa (2020, p. 150), "entendemos que os itinerários terapêuticos se produzem na intersecção de uma história singular situada em determinado contexto histórico e social". Por meio do grupo, buscou-se traçar os itinerários terapêuticos de modo a tornar mais conscientes tanto as variadas determinações e consequências do processo de sofrimento psíquico quanto o papel dos serviços de saúde, apontando para uma crítica ao reducionismo imposto pelo modelo biomédico. Consideramos que a exclusão e o enclausuramento exacerbam o sofrimento psíquico dos sujeitos, sendo fundamental a reinserção social, fomentando a importância do convívio com a sociedade e reiterando a importância da ocupação cidadã dos espaços sociais (Paranhos-Passos \& Aires, 2013; Pitta, 1996). Dessa forma, os conceitos de contratualidade e reabilitação psicossocial, abordados por Kinoshita (1996) e Pitta (1996), se mostram fundamentais para a atuação no CAPS, já que é papel da equipe promover as mediações necessárias para restituir o poder contratual da/o usuária/o, ou seja, criar as condições necessárias para que esses sujeitos possam participar do processo de trocas sociais, aumentando a autonomia e promovendo a reinserção social. O grupo permitiu desenvolver essas trocas por meio da construção, junto às/aos usuárias/os, de novas possibilidades de pensar a própria identidade e do desenvolvimento de novos interesses e atividades para além do CAPS.

\section{Considerações Finais}

Por meio do desenvolvimento do grupo foi possível identificar que os itinerários terapêuticos, ou seja, a busca de cuidados em saúde mental de alguns dos participantes do grupo teve início anterior à criação dos CAPS, quando os indivíduos passaram por internações psiquiátricas ou foram assistidos em clínicas conveniadas de psicologia e psiquiatria. No entanto, notase que foi apenas após a criação do CAPS que as/os usuárias/os passaram a receber um cuidado em saúde mental por equipes multiprofissionais e orientado para a elaboração de projetos e para uma vida mais autônoma.

A atual política de saúde mental prevê que o cuidado às pessoas em sofrimento psíquico seja ofertado na Rede de Atenção Psicossocial (RAPS), entretanto foi observado que a atenção às pessoas em sofrimento psíquico crônico ou agravado, como é o caso das/os participantes do grupo, acaba muitas vezes restrita a um único serviço: o Centro de Atenção Psicossocial. O CAPS é o serviço que presta atendimento de saúde mental multiprofissional e especializado e tem como objetivo promover a reinserção social e reabilitação psicossocial. Ainda que seja, portanto, um dispositivo importante da RAPS, não tem como finalidade atender a todas as necessidades (mesmo que apenas as necessidades em saúde) de seus usuários e usuárias. A política de saúde mental, ao propor o cuidado em rede, defende um cuidado integral e contínuo. O desenho da RAPS garante atenção psicossocial especializada, mas também que a/o usuária/o não deixe de ser acompanhado pelos outros pontos da rede, conforme suas necessidades, como a atenção básica, ambulatório de saúde mental, centros de convivência e cultura e demais equipamentos da rede de saúde e da rede intersetorial.

A intervenção no Grupo Trilhas, ao permitir o debate sobre a RAPS e o conhecimento dos dispositivos e possibilidades do território, favoreceu a busca por estes outros serviços, por recursos do território e estratégias terapêuticas, promovendo autonomia e ampliação das trocas sociais dos participantes. Dessa forma, pensar os itinerários terapêuticos no contexto das 
pessoas em sofrimento psíquico auxilia os indivíduos a perceberem e significarem o próprio sofrimento, a encontrar o suporte adequado em relação à sua saúde e a compreenderem a relação do adoecimento com as suas histórias de vida. Portanto, considerando suas repercussões sobre os itinerários terapêuticos de usuárias/os, é fundamental evidenciar as conquistas (bem como reconhecer os limites) da reforma psiquiátrica e posicionar-se em defesa de seu avanço na direção de um cuidado em liberdade e de um projeto antimanicomial, em especial nesse momento de importantes retrocessos no campo da saúde mental.

\section{Referências}

Almeida, M. R. A formação social dos transtornos do humor. [Tese]. Botucatu: Universidade Estadual Paulista "Júlio de Mesquita Filho"; 415 p.

Alves, P C. (2015) Itinerário terapêutico e os nexus de significado da doença. Rev Ciênc Soc 42: 29-43.

Amarante, P. (1995) Loucos pela vida: a trajetória da reforma psiquiátrica no Brasil. Fiocruz

Amarante, P. (2007) Saúde mental e atenção psicossocial. Editora Fiocruz.

Amarante, P.; \& Nunes, M. O. (2018) A reforma psiquiátrica no SUS e a luta por uma sociedade sem manicômios. Ciênc. saúde coletiva, 23(6), 2067-2074, https://doi.org/10.1590/1413-81232018236.07082018.

Andrade, M. C., Burali, M. A. D. M., Vida, A., Fransozio, M. B. B., \& Santos, R. Z. D. (2013). Loucura e trabalho no encontro entre saúde mental e economia solidária. Psicologia: ciência e profissão, 33, 174-191.

Azevedo, D. M. D., \& Miranda, F. A. N. D. (2011). Oficinas terapêuticas como instrumento de reabilitação psicossocial: percepção de familiares. Escola Anna Nery, 15, 339-345. https://doi.org/10.1590/S1414-81452011000200017

Brasil (2001) Lei n. ${ }^{\circ}$ 10216, de 06 de abril de 2001. Dispõe sobre a proteção e os direitos das pessoas portadoras de transtornos mentais e redireciona o modelo assistencial em saúde mental 2004; 5: 17-19.

Brasil (2011). Portaria n 3.088 , de 23 de dezembro de 2011. Rede de Atenção Psicossocial para pessoas com sofrimento mental ou necessidades decorrentes do uso de álcool e outras drogas. Diário Oficial da União 23 de dezembro de 2011.

Brasil (2017) Portaria de Consolidação n 3, de 03 de outubro de 2017. http://bvsms.saude.gov.br/bvs/saudelegis/gm/2017/prc0003_03_10_2017. html.

Buss, P. M., \& Filho A. P, (2007). A saúde e os determinantes sociais, PHYSIS: Rev. Saúde Coletiva, 17(1):77-93, https://doi.org/10.1590/S010373312007000100006

Costa, L. A. Determinações sociais de itinerários terapêuticos produtores de cuidado e de saúde em situações de sofrimento psíquico: o papel do Centro de Atenção Psicossocial (CAPS). [Dissertação]. Campo Grande-MS: Fundação Universidade Federal de Mato Grosso do Sul; 170 p

Campos, R. T., Passos, E., \& Palombini, A. (2014). Gestão Autônoma da Medicação - Guia de Apoio a Moderadores. https://www.fcm.unicamp.br/fcm/sites/default/files/paganex/guia_gam_moderador_-_versao_para_download_julho_2014.pdf

Gerhardt, T. E. (2006). Itinerários terapêuticos em situações de pobreza: diversidade e pluralidade. Cadernos de Saúde Pública, 22(11), 2449-2463. https://doi.org/10.1590/S0102-311X2006001100019

Kinoshita R. T. (1996). Contratualidade e reabilitação psicossocial. In: Pitta A, organizadores. Reabilitação psicossocial no Brasil. Hucitec

Lane, S. T. (1984). O processo grupal. In Lane, S. T e Codo W. Organizadores. Psicologia social - o homem em movimento. $78-98$.

Lappann-Botti, N. C., \& Labate, R. C. (2004) Oficinas em saúde mental: a representação dos usuários dos serviços de saúde mental. Texto \& Contexto Enferm. out/dez; 13(4): 519-26 https://doi.org/10.1590/S0104-07072004000400003

Leontiev, A. N. (1969) Las necesidades y los motivos de la actividad. In: Smirnov A, Leontiev, AN. Psicologia. Grijalbo. $341-354$.

Martins, L. M. (2007) A formação social da personalidade do professor. Autores Associados.

Oliveira, E. N., \& Jorge, M. S. B. (2007) Violência contra mulher: sofrimento psíquico e adoecimento mental, Rev. RENE. 8(2), 93-100.

Pande, M. N. R., \& Amarante, P. D. C. Desafios para os Centros de Atenção Psicossocial como serviços substitutivos: a nova cronicidade em questão. Ciência \& Saúde Coletiva. 16(4), 2067-2076. <https://doi.org/10.1590/S1413-81232011000400006>.

Paranhos-Passos, F., \& Aires, S. (2013). Reinserção social de portadores de sofrimento psíquico: o olhar de usuários de um Centro de Atenção Psicossocial. Physis: Revista de Saúde Coletiva, 23, 13-31. https://doi.org/10.1590/S0103-73312013000100002

Pitta, A. (1996) O que é reabilitação psicossocial no Brasil hoje? In Pitta. A Reabilitação Psicossocial no Brasil. Hucitec

Santos, A. M. C. C. (2009) Articular saúde mental e relações de gênero: dar voz aos sujeitos silenciados. Ciência \& Saúde Coletiva. 14(4)1177-1182. $<$ https://doi.org/10.1590/S1413-81232009000400023>. 
Research, Society and Development, v. 10, n. 14, e126101421663, 2021

(CC BY 4.0) | ISSN 2525-3409 | DOI: http://dx.doi.org/10.33448/rsd-v10i14.21663

Souza, F. F., \& Zambenedetti, G. (2018). Percursos de cuidado: pistas sobre itinerários terapêuticos em saúde mental. Tempus Actas de Saúde Coletiva, 11(4), 105. http://dx.doi.org/10.18569/tempus.v11i4.2485

Tenório, F. (2002). A reforma psiquiátrica brasileira, da década de 1980 aos dias atuais: história e conceitos. História, Ciências, Saúde-Manguinhos, 9, 25-59. https://doi.org/10.1590/S0104-59702002000100003. 